\title{
Molecular Orbital Calculation on 1.2-Difuryl- and 1.2-Dithienyl-ethylenes and Their Photocyclisation Products the Benzodifuranes and Benzodithiophenes
}

\author{
H. Güsten, L. Klasinc * and O. Volkert ** \\ Institut für Strahlenchemie, Kernforschungszentrum Karlsruhe \\ (Z. Naturforschg. 24 b, 12-15 [1969] ; eingegangen am 26. März 1968)
}

\begin{abstract}
Es werden die HMO-Berechnungen der 1.2-Difuryl- und 1.2-Dithienyl-äthylene sowie die bei der photochemischen Cyclodehydrierung dieser Verbindungen entstehenden Benzodifurane und Benzodithiophene mitgeteilt. Die chemische Reaktivität dieser Verbindungen wird an Hand der berechneten Reaktivitätsindices vorausgesagt.
\end{abstract}

During the last year a series of publications has 1,2-dithienyl-ethylenes ${ }^{1-3}$. These compounds underdealt with the photochemistry of 1,2-difuryl- and go photochemical cis-trans isomerization ${ }^{1}$ as well as

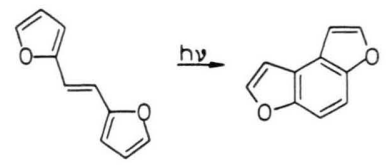

1,2-Di-(2,3'-furyl) -ethylene

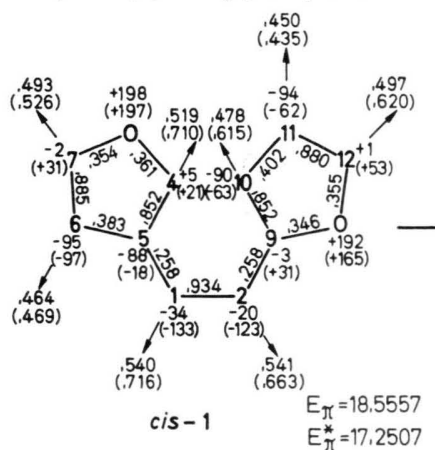

Benzo[1,2-b:3,4-b']-difuran

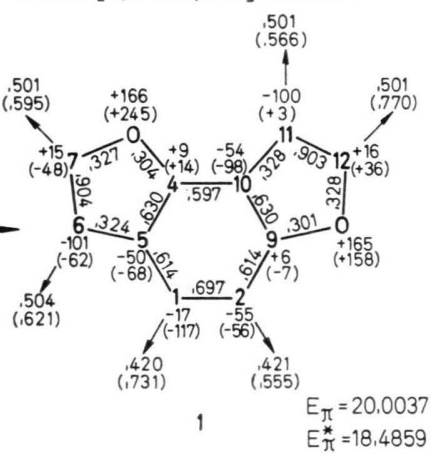

$E_{\pi}=$ total $\pi$-energy for the ground state. $E_{\pi}{ }^{*}=$ total $\pi$-energy for the excited state.

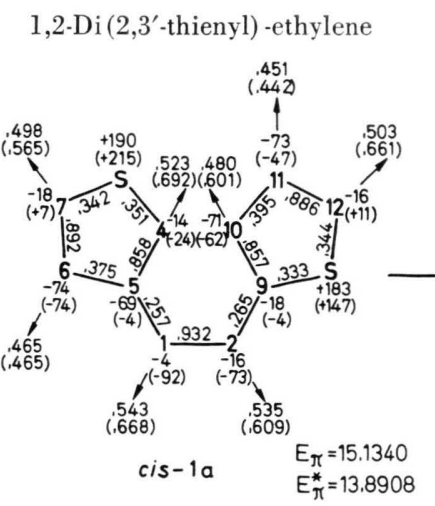

* On leave from institute "Ruđer Bošković", Zagreb, Yugoslavia.

* Present address: Badische Anilin- und Soda-Fabrik, Ludwigshafen.

1 C. M. Orlando, A. A. Zimmerman, M. H. Gianni, and K. $\mathrm{W}_{\mathrm{EISS}}$, J. org. Chemistry, in press.
Benzo [1,2-b:3,4-b'] -dithiophene

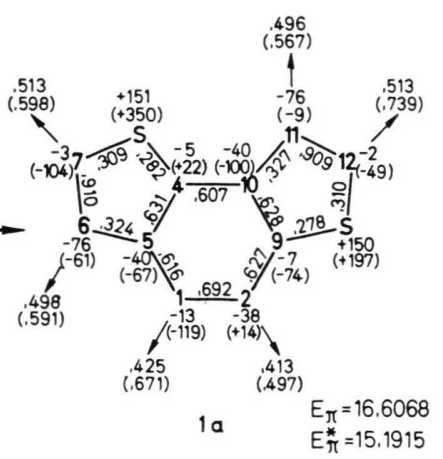

2 R. M. Kellogg, M. B. Groen, and H. W ynberg, J. org. Chemistry 32, 3093 [1967] ; H. W ynberg and M. B. Groen, J. Amer. chem. Soc. 90, 5339 [1968].

3 C. E. Loader and C. J. Timmons, J. chem. Soc. [London] (C) 1967,1677 . 
1,2-Di-(3-furyl) -ethylene

Benzo[1,2-b :4,3-b']-difuran
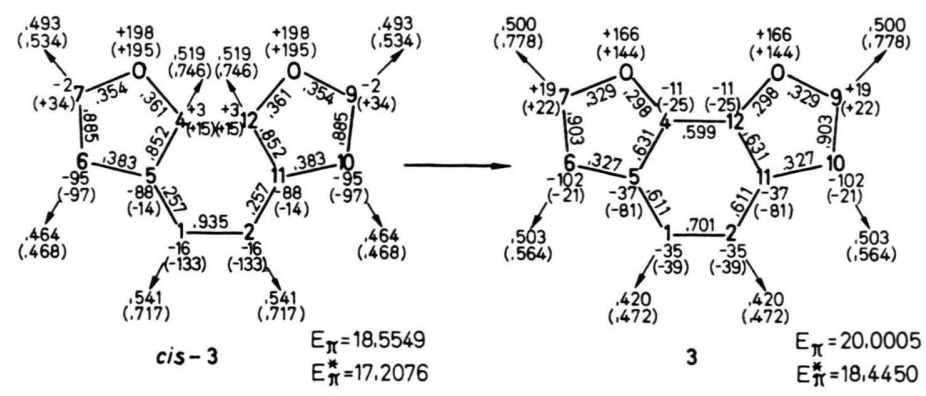

1,3-Di-(2-thienyl) -ethylene

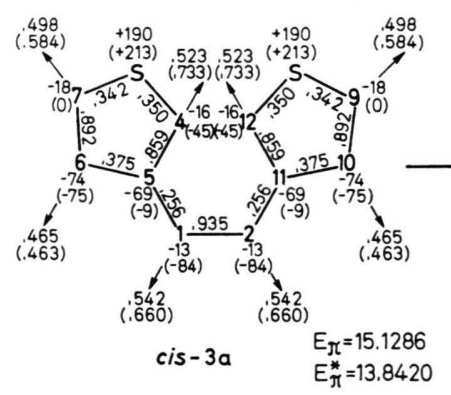

Benzo [2,1-b :3,4-b']-dithiophene

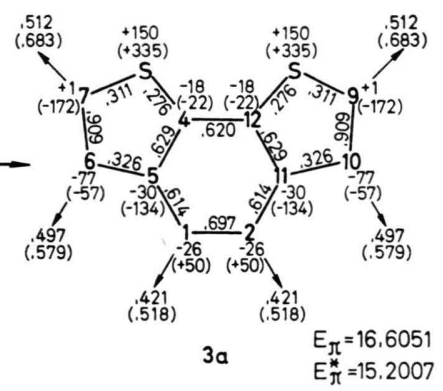

1,2-Di-(2-furyl) -ethylene

Benzo [1,2-b :4,3-b']-difuran
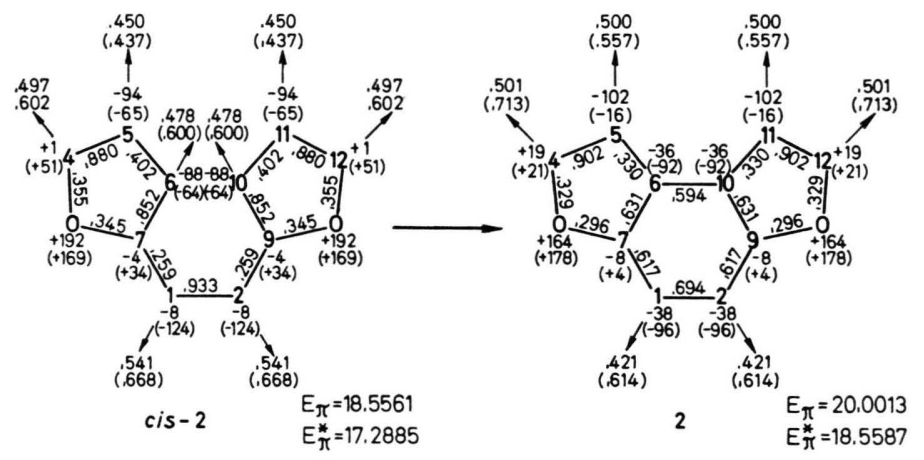

1,2-Di-(2-thienyl) -ethylene

Benzo [1,2-b :4,3-b']-dithiophene
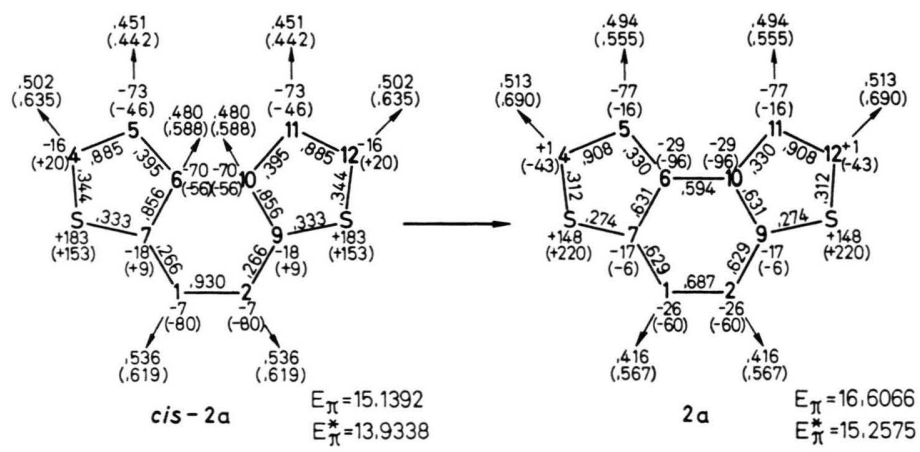
photocyclisation ${ }^{2,3}$. In presence of oxidizing agents 1,2-dithienyl-ethylenes yield benzodithiophenes ${ }^{2}$. Similarly 1,2-di-(2-furyl)-ethylene results benzo[ $[1,2$ $\left.\mathrm{b}: 4,3-\mathrm{b}^{\prime}\right]$-difuran ${ }^{3}$.

By means of this photocyclodehydrogenation the unsubstituted compounds of benzodifuranes and benzodithiophenes which besides the benzo[1,2-b: 4,3-b']-dithiophene ${ }^{4}$ are unknown, are now available.

The present communication reports a theoretical study of some 1,2-difuryl- and 1,2-dithienyl-ethylenes in order to get some information about the mechanism of the photocyclisation. Furthermore we report the reactivity indices of the benzodifuranes and benzodithiophenes regarding substitution reactions as to predict the positions of electrophilic, nucleophilic and radical attack.

\section{Theoretical Approach}

In general the photocyclisation of 1,2-difuryland 1,2-dithienyl-ethylenes produces six possible isomers of benzodifuran as well as benzodithiophenes. From chemical argumentations accompanying the photocyclisation only the following isomers should be stable compounds: benzo[1,2-b:3,4-b']difuran (1) and -dithiophene (1 a), benzo[1,2-b: 4,3-b']-difuran (2) and -dithiophene $(\mathbf{2} \mathbf{a})$ and the

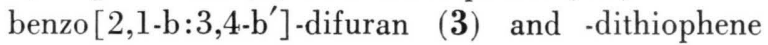
$(3 \mathbf{a})$. To these six molecules and the corresponding three difuryl- and dithienyl-ethylenes we applied the MO-theory within the framework of the $\mathrm{Hückel}$ method ${ }^{5}$. The assumption was made that all investigated molecules are planar. The empirical parameters used in the calculation are shown in table I.
A similar choise of parameter has been given by Sappenfield and Kreevoy ${ }^{6}$ for furan and thiophene. We calculated the $\pi$-bond orders, the $\pi$-electron densities, the free valence indices and the total $\pi$-energy of the molecules for the ground and the excited state. The results are presented in the molecular diagrams showing the starting 1,2-disubstituted ethylene and the resulting photocyclodehydrogenation product. All numbers in brackets refer to the excited state. The charge densities are multiplied by 1000 . The numbers at the end of the arrows represent the free valence indices. For the seek of simplicity the numbering of the atoms in our skeletal structures is somewhat different. We always count the ethylenic carbon atoms in the difuryl- and dithienyl-ethylenes as 1 and 2 , the heteroatom in the rings as 3 and 8 respectively, and the numbering of the remaining ring carbon atoms is going clockwise upward. The same numbering is applied to the photocyclodehydrogenation product.

\section{Discussion}

The mechanism of the photocyclisation reaction of cis-stilbenes is not completely cleared as yet ${ }^{7-9}$. Several authors assume that the photochemical part of the reaction proceeds from the excited state of the cis-compound to the ground state of the cyclisation product ${ }^{7-9}$. An other discussed mechanism suggests that the photochemical ring closure proceeds via vibrationally excited ground state levels of the cis-compound which are reached in a fast $\mathrm{S}_{1} \rightarrow \mathrm{S}_{0}$-deactivation process from the short living excited singlet state of the cis-compound ( $\mathrm{Lew}$ is mechanism $)^{10}$. In a study of the photocyclisation

\begin{tabular}{lrrrrr}
\hline & \multicolumn{2}{c}{ Ethylene-1,2-di- } & & \multicolumn{2}{c}{ Benzodi- } \\
& -furanes & -thiophenes & -furanes & -thiophenes \\
\hline$\alpha_{1}$ & $\alpha+0.02 \beta$ & $\alpha+0.02 \beta$ & $\alpha$ & $\alpha$ \\
$\alpha_{\mathrm{x}}{ }^{*}$ & $\alpha+1.72 \beta$ & $\alpha+0.93 \beta$ & $\alpha+1.72 \beta$ & $\alpha+0.93 \beta$ \\
$\alpha_{4}$ & $\alpha$ & $\alpha$ & $\alpha$ & $\alpha$ \\
$\alpha_{5}$ & $\alpha-0.04 \beta$ & $\alpha-0.02 \beta$ & $\alpha-0.04 \beta$ & $\alpha-0.02 \beta$ \\
$\beta_{12}$ & $1.02 \beta$ & $1.02 \beta$ & All resonance integrals in the \\
$\beta_{1 \text { (ring }}$ & $0.54 \beta$ & $0.60 \beta$ & resulting benzene ring are equal to $\beta$ \\
$\beta_{34}$ & $0.76 \beta$ & $0.50 \beta$ & $0.76 \beta$ & $0.50 \beta$ \\
$\beta_{56}$ & $0.59 \beta$ & $0.64 \beta$ & $0.59 \beta$ & $0.64 \beta$ \\
\hline
\end{tabular}

Table I. Resonance and Coulomb Integrals. ${ }^{*} \mathrm{x}=$ heteroatom.

${ }^{4}$ D. S. RAO and B. D. TILAK, J. Sci. ind. Res. [New Delhi] Sect. B 16, 65 [1957]; C. A. 51, 13841 [1957].

5 E. Hǘckel, Z. Physik 70, 204 [1931].

${ }^{6}$ D. S. Sappenfield and M. Kreevoy, Tetrahedron [London] 19 (Suppl. 2), 157 [1963].

7 F. R. Stermitz, in: O. L. Chapman's "Organic Photochemistry I”, M. Dekker Inc., New York 1967, p. 247.
8 K. A. Muszkat and E. Fischer, J. chem. Soc. [London] (B) 1967,662 .

9 M. Scholz, F. Dietz, and M. Mühlstädt, Z. Chem. 7, 329 [1967].

10 H. Güsten and L. Klasinc, Tetrahedron [London] 24, 5499 [1968]. 
of para- and meta-substituted cis-stilbenes we were able to show that the quantum yield of the photocyclisation ${ }^{11}$ is going linear with a reactivity index $q_{\mathrm{rs}}$, being the product of the electron densities of the reacting $o, o^{\prime}$-positions in the ground state ${ }^{10}$. Applying this criterion to the photocyclisation of the difuryl- and dithienyl-ethylenes i.e. multiplying the excited and ground state $\pi$-electron densities of the reacting positions we obtain the following results.

\begin{tabular}{lllllll}
\hline & cis-1* & cis-1 a & cis-2 & cis-2a & cis-3 & cis-3a \\
\hline$q_{\mathrm{rs}}^{*}$ & 1.041 & 1.087 & 1.132 & 1.115 & 0.970 & 1.092 \\
$q_{\mathrm{rs}}$ & 1.085 & 1.086 & 1.184 & 1.145 & 0.994 & 1.032
\end{tabular}

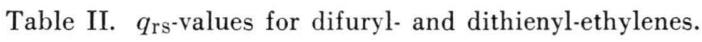

The $q_{\mathrm{rs}}^{*}$ - and $q_{\mathrm{rs}}$-values predict a photocyclisation probability in the order of cis-2 $>c i s-\mathbf{2} \mathbf{a}>c i s-$ $\mathbf{3} \mathbf{a}>c i s-\mathbf{1} \mathbf{a}>c i s-\mathbf{1}>c i s-\mathbf{3}$ and $c i s-\mathbf{2}>c i s-$ $\mathbf{2} \mathbf{a}>c i s-\mathbf{1} \mathbf{a}>c i s-\mathbf{1}>c i s-\mathbf{3} \mathbf{a}>c i s-\mathbf{3}$, respectivly. According to the experimental data ${ }^{2,3}$ the $c i s$ 2-, cis-1 a, cis-2 a- and cis-3 a-compounds undergo photocyclisation. With the exception of $\mathbf{3} \mathbf{a}$ the oxidation products 2 a $(90 \%), 2(24 \%)$ and 1 a $(47 \%)$ have been isolated ${ }^{2,3}$ (yield in parenthesis). In cis$3 \mathbf{a}$ the abstraction of hydrogen leeds to a $\beta$-thiosubstituted radical which undergoes very rapid elimination of the sulfur as thiyl radical ${ }^{* *}$. From our theoretical data we can expect that 1 can be obtained by photocyclodehydrogenation and $\mathbf{3}$ probably not. On the contrary to the HMO-calculations for the photocyclisation of mono-substituted cisstilbenes ${ }^{10}$ the results of the HMO-calculation for the photocyclisation of 1,2-difuryl- and dithienylethylenes give no distinction whether the photocyclisation proceeds via the excited or the ground state of the cis-compound. Although the predicted

11 H. Jungmann, H. Güsten, and D. Schulte-Frohlinde, Chem. Ber. 101, 2690 [1968].

* cis-1 is the corresponding 1,2-disubstituted ethylene yielding 1 on photocyclodehydrogenation.

* see $^{2}$ and papers cited there.

12 J. N. Murrell, S. F. A. Kettre, and J. M. Tedder, "Valence Theory", John Wiley, London 1965, p. 317.

13 Derivatives of benzo[1,2-b:3,4-b']-difuran: T. Tominaga, J. pharmac. Soc. Japan [Yakugakuzasshi] 76, 1385 [1956]; G. R. Kelkar and D. B. Limaye, Rasayanam [J. Progr. chem. Sci.] 1, 228 [1943] ; C. A. 36, 1037 [1942] ; D. B. Limaye and V. V. Nagarkar, ibid. 1, 255 [1943] ; C. A. 38, 4264 [1944].

14 Derivatives of benzo[1,2-b:4,3-b']-difuran: R. Royer, E. Bisagni, A. M. Latal-Jeantet, and J. R. Marquet, Bull. Soc. chim. France 1965, 2607; R. Royer, E. Bisagni, C. order of the $q_{\mathrm{rs}}$-values (ground state) fits better the experimental data than the $q_{\mathrm{rs}}^{*}$-values, we feel that the small differences between the $q_{\mathrm{rs}^{-}}$and $q_{\mathrm{rs}}^{*}$ values do not permit a discrimination on the mechanism of the photocyclisation.

In predicting the chemical reactivities shown in table III we have followed the isolated molecule approximation ${ }^{12}$ from which we can expect a correla-

\begin{tabular}{clll}
\hline Compound & Electrophilic & Nucleophilic & \multicolumn{1}{c}{ Radical } \\
\hline $\mathbf{1}$ & $6,11>2 *$ & $12,7>1$ & $6 \geqq 7,11,12$ \\
$\mathbf{2}$ & $5,11>1,2$ & $4,12>1,2$ & $4,12 \geqq 5,11$ \\
$\mathbf{3}$ & $6,10>1,2$ & $7,9>1,2$ & $6,10 \geqq 7,9$ \\
$\mathbf{1} a$ & $6,11>2$ & $12 \geqq 7>1$ & $12,7>6$ \\
$\mathbf{2} a$ & $5,11>1,2$ & $4,12>1,2$ & $4,12>5,11$ \\
$\mathbf{3} \mathrm{a}$ & $6,10>1,2$ & $7,9>1,2$ & $7,9>6,10$ \\
cis-1 & $6 \geqq 11>10$ & $4>12>7$ & $1,2>4$ \\
cis-2 & $5,11>6,10$ & $4,12>1,2$ & $1,2>4,12$ \\
cis-3 & $6,10>1,2$ & $4,12>7,9$ & $1,2>4,12$ \\
cis-1a & $6 \geqq 11>10$ & $1>4>2,12$ & $1>2>4$ \\
cis-2a & $5,11>6,10$ & $1,2>4,12$ & $1,2>4,12$ \\
cis-3a & $6,10>7,9$ & $1,2>4,12$ & $1,2>4,12$ \\
\hline
\end{tabular}

Table III. The Prediction of Chemical Reactivity Based on Charge Densities and Free Valence Indices. * The numbers refer to the numbers in the skeletal structures.

tion between the electron densities and the attacking charged species. For radicals the correlation with the free valence proved to be successful. The most reactive position in benzodifuranes and benzodithiophenes is generally in the five membered rings. In the difuryl- and dithienyl-ethylenes this position tends to be on the ethylenic double bond (especially for radical attack). Although not much synthesis has been done on these molecules as yet the available results support our predictions ${ }^{4,13-17}$.

We thank Priv.Doz. Dr. D. Schulte-Frohlinde for his support of this work.

Hudry, A. Chentin, and M. L. Desvoye, Bull. Soc. chim. France 1963, 1003; R. Pummerer, E. Buchta, E. Deimler, and E. Singer, Chem. Ber. 75 B, 1976 [1942] ; O. DischenDORFER, Mh. Chem. 66, 201 [1935].

15 Derivatives of benzo[2,1-b:3,4-b']-difuran: R. Royer, J. L. Derocque, P. Demerseman, and A. Chentin, C. R. Ser. C, 262, 1286 [1966]; W. Limontschew and E. Wiesenberger, Mh. Chem. 83, 137 [1952]; O. Dischendorfer and W. LiMONTSCHEW, ibid. 80, 741 [1949].

16 Derivatives of benzo[1,2-b:3,4-b']-dithiophene: D. S. RAO and B. D. TIlak, J. sci. ind. Res. [New Delhi] Sect. B 13, 829 [1954] ; C. A. 50, 934 [1956].

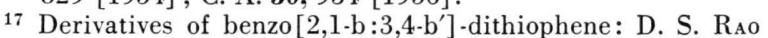
and B. D. Tilak, J. sci. ind. Res. [New Delhi] Sect. B 17, 260 [1958] ; C. A. 55, 22282 [1961]. 